\title{
Hospital readmission rates following implementation of an antimicrobial stewardship intervention in acute inpatient psychiatric units
}

\author{
Abdulhamid A Althagafi ${ }^{1,2 *}$, Mohannad Alshibani ${ }^{1,2}$, Saad Fallatah ${ }^{2,3}$ and Georgina Rubal-Peace ${ }^{4}$ \\ ${ }^{1}$ Clinical Pharmacy Department, College of Pharmacy, King Abdulaziz University, Jeddah, Saudi Arabia \\ ${ }^{2}$ Center for Health Outcomes and PharmacoEconomic Research, University of Arizona, Tucson, AZ, USA \\ ${ }^{3}$ Clinical and Hospital Pharmacy Department, College of Pharmacy, Taibah University, Medina, Saudi Arabia \\ ${ }^{4}$ Banner University Medical Center South, Tucson, AZ, USA
}

\begin{abstract}
Objective: Hospital readmission represent a significant cost burden for healthcare systems. The presence of mental health disorders can increase the likelihood of readmission, especially in patients with co-morbidities. The presence of infection also increases the likelihood of readmission. Data regarding the effect of antimicrobial stewardship on readmission rates are conflicting. The aim of this study is to compare the rate of all-cause 30-day readmission in patients admitted to an acute inpatient psychiatric unit before and after implementation of a pharmacist-led antimicrobial stewardship intervention.
\end{abstract}

Methods: This study was a retrospective chart review. Patients were included if they were 18 years or older, had been admitted to an inpatient adult acute psychiatric unit, and had received at least one antimicrobial medication. An antimicrobial stewardship intervention was implemented on August 1, 2014, in hospital in Tucson, Arizona. Data from the period of August 1, 2013, to July 1, 2014, represented the pre-intervention group, and data collected from the period of August 1, 2014, to August 1, 2015, represented the post-intervention arm.

Results: There were a total of 200 patients in the study. There were no significant differences between the two groups in terms of all-cause readmission and infectionrelated readmission within 30 days. However, our study found that there was a significantly shorter length of hospital stay in the post-intervention group compared to the pre-intervention group ( $6.6 \pm 4.4$ vs $9.0 \pm 6.5$ days, respectively; $P=0.003$ ). Cephalosporin use was significantly lower in the post-intervention group compared to the pre-intervention group ( 24 vs 44 , respectively; $P=0.015$ ).

Conclusions: Among patients admitted to an acute inpatient psychiatric unit, a pharmacist-led antimicrobial stewardship intervention has minimal effect on 30-day hospital readmission. However, it was associated with a significant shorter length of stay.

\section{Introduction}

\section{Background}

Hospital readmissions are responsible for a large proportion of healthcare costs in the United States, leading hospital administrators to seek ways of reducing readmission rates. Among Medicare enrollees, the readmission rate is about $19 \%$ within 30 days of discharge [1]. Readmission rates are also high in patients with psychiatric conditions, which can complicate the management of concurrent infections. One study has suggested that the rate of infections in patients with mental health disorders is high; however, it is difficult to assess the significance of this evidence due to the lack of a comparative group [2]. Other studies have identified a potential association between psychiatric disorders and increased 30-day readmissions for other medical conditions $[3,4]$.

Currently, evidence of the relationship between pharmacist-led antimicrobial stewardship and readmission rates is conflicting. One study has demonstrated that implementing antimicrobial stewardship was associated with a lower rate of readmission due to unresolved infections [5]. However, other studies have shown that there is no effect of such an intervention on 30-day hospital readmission rates [6,7].

The purpose of this study is to compare the rate of all-cause 30-day readmission in patients admitted to an acute inpatient psychiatric unit at an academic medical center before and after implementation of an antimicrobial stewardship intervention led by pharmacist.

\section{Methods}

\section{Study design}

This was a retrospective observational study conducted between August 1, 2013, and August 31, 2015. The study was conducted in an academic medical center affiliated with the University of Arizona with 245 beds. It has two adult acute inpatient behavioral health units with 48 beds and one geriatric acute inpatient behavioral health unit with 18 beds. The study included all patients in the adult acute inpatient

Correspondence to: Abdulhamid A Althagafi, Pharm D, Postdoctoral Pharmacy Fellow, Center for Health Outcomes and Pharmaco Economic Research, The University of Arizona-College of Pharmacy, USA, Tel: 5203038681; Fax: 5206267355; E-mail: Althaghf@pharmacy.arizona.edu

Key words: antimicrobial stewardship, pharmacist intervention, psychiatric, readmission

Received: November 09, 2017; Accepted: November 30, 2017; Published: December 04, 2017 
behavioral health units. Institutional review board approval was obtained prior to conducting the study.

\section{Study population}

Patients were included in the study if they met the following criteria: (1) were aged 18 years or older, (2) were admitted to an inpatient adult acute psychiatric unit at Banner-University Medical Center South (BUMCS) between August 1, 2013, and August 31, 2015, and (3) received at least one antibacterial medication. Eligible patients were identified through the use of the hospital's electronic database. Patients who were admitted to behavioral health units but did not receive an antibiotic during their stay were excluded. We conducted a propensity-score matched cohort analysis of eligible individuals who were discharged from August 1, 2013 through July 31, 2014 (preintervention group) and discharged between August 1, 2014, and July 31,2015 (post-intervention group). We randomly selected 100 patients in each group to provide us with sufficient descriptive information.

\section{Intervention}

The antimicrobial stewardship intervention at BUMCS began in July 2014 as a new service to improve antimicrobial use and overall care. The intervention was initiated as part of an expanded pharmacy services program and was not started for the purpose of this research study. Eligible subjects discharged from August 1, 2013, through July 31,2014 , were included in the pre-intervention group, and eligible subjects discharged from August 1, 2014, through July 31, 2015, were included in the post-intervention group.

As a part of the intervention, all hospitalized patients in the behavioral health units who received antibiotics were followed by pharmacy services starting on July 1, 2014. A weekly report was generated that detailed the antibiotics prescribed in the behavioral health unit. Furthermore, during each shift, a designated pharmacist was responsible for following up with each patient who received antibiotics. The designated pharmacist evaluated appropriateness based on the choice of antibiotic for indication or cultures, antibiotic dose for indication, dose adjustment in patients with renal impairment, and duration of antibiotic course. The assessment of appropriateness was based on Infectious Diseases Society of America (IDSA) guidelines, the Johns Hopkins Guide for Antibiotics, and the Lexicomp drug information reference [8-10]. If an antibiotic therapy were deemed inappropriate, the pharmacist communicated with the prescribing physician to make antibiotic adjustment recommendations and documented the intervention on the patient's chart.

\section{Outcomes}

The primary endpoint of this study was all-cause readmission at 30 days after discharge. The secondary endpoints were any infectionrelated readmission at 30 days and hospital length of stay.

The data collection consisted of patient age, sex, race, ethnicity, psychiatric diagnosis at discharge, antibiotic used, length of hospital stay for both admission and readmission, type of antibiotic prescription upon discharge from the first admission, and type for readmission, if applicable. Type of readmission was categorized into all-causes readmission or infection related readmission. Data collection was performed by retrospective chart review onsite at BUMCS using electronic health records identified from the hospital electronic database.

For statistical analysis, the outcomes of all-cause readmission and infection-related readmission within 30 days were evaluated using a chi square test. The secondary outcome of hospital length of stay was assessed using unpaired Student's t test. Also, the unpaired Student's $t$ test was used to compare all normally distributed continuous variables. Demographic characteristics including categorical variables were assessed using a chi-square analysis. We could not calculate power size for our project due to the lack of similar studies. For each group, randomization was performed using a computer-based random number generator, which created a "pseudo-number generator" by using a complex algorithm. From the pool of eligible patients, we randomly selected 100 patients for each group. The a-priori level of significance used for all analyses was $P<0.05$.

\section{Results}

The demographic characteristics for the pre-intervention and post-intervention groups are shown in Table 1. The mean ages of the pre-intervention and post-intervention groups were $43.2 \pm 11.9$ vs $42.2 \pm 12.9$ years; $P=0.526$, respectively. The pre-intervention and postintervention groups were well-matched for sex, race, and ethnicity (Table 1).

The pre-intervention and post-intervention groups were similar in the following categories: psychotic disorders, bipolar disorders, mood disorders, and depressive disorders. Antimicrobial use showed no significant differences between the pre-intervention and postintervention groups except that the use of cephalosporin was lower in the post-intervention group compared to the pre-intervention group ( 24 vs 44 , respectively; $P=0.015$ ). In contrast, there was an increase in the use of fluoroquinolones (ciprofloxacin and levofloxacin) in the post-intervention group than in the pre-intervention group (17 vs 8 , respectively; $P=0.071$ ) (Table 1 ).

\section{Main results}

For the primary outcome, all-cause readmissions within 30 days, there was no significant difference between the pre-and postintervention groups ( 28 vs $28 ; P=1)$. Also, there was no significant difference between the two groups in infection-related readmissions within 30 days ( 2 vs $4 ; P=0.414)$. However, our study found that there was a significantly shorter length of hospital stay in the postintervention group compared to the pre-intervention group (6.6 \pm 4.4 vs $9.0 \pm 6.5$ days, respectively; $P=0.003$ ) (Table 2).

\section{Discussion}

To the best of our knowledge, this is the first study to focus on the effect of implementing a pharmacist-led antimicrobial intervention on the readmission rate in patients admitted to behavioral health units. Nevertheless, we found no significant differences between groups for all-cause readmission and infection-related readmission within 30 days. Our findings are supported by other previous studies that show implementation of antimicrobial stewardship has minimal effect on readmission rates $[3,4]$.

A major finding in the current study was the statistically significant shorter length of hospital stay in the post-intervention group. In patients admitted to behavioral health units who were diagnosed with mental health disorders and prescribed at least one oral antimicrobial in the period of post-intervention, we found that the average length of stay was 6.6 days, compared to 9.0 days for pre-intervention group. This finding may suggest that pharmacist-led antimicrobial intervention is related to a shorter length of stay in patients diagnosed with mental health disorders. This finding is supported by Zhou, et al. [11] who found that pharmacist-led antimicrobial stewardship in patients 
Table 1. Baseline patient characteristics

\begin{tabular}{|c|c|c|c|}
\hline Demographics & Pre-intervention $(\mathrm{N}=100)$ & Post-intervention $(\mathrm{N}=100)$ & $P$ value \\
\hline Age, (mean, $\pm \mathrm{SD})$ & $43.2 \pm 11.9$ & $42.2 \pm 12.9$ & 0.526 \\
\hline Female, n (\%) & $63(63)$ & $66,(66)$ & 0.791 \\
\hline White, n (\%) & $86,(86)$ & $83,(83)$ & 0.817 \\
\hline Non-Hispanic, n (\%) & $85,(85)$ & $87,(87)$ & 0.705 \\
\hline Hispanic, n (\%) & $15,(15)$ & $13,(13)$ & 0.705 \\
\hline $\begin{array}{l}\text { Diagnostic category, n (\%) } \\
\text { Psychotic disorders } \\
\text { Bipolar disorders } \\
\text { Mood disorders } \\
\text { Depressive disorders } \\
\text { Other }\end{array}$ & $\begin{array}{l}40,(40) \\
24,(24) \\
17,(17) \\
16,(16) \\
3,(3)\end{array}$ & $\begin{array}{l}32,(32) \\
24,(24) \\
16,(16) \\
15,(15) \\
13,(13)\end{array}$ & $\begin{array}{l}0.345 \\
1 \\
0.861 \\
0.857 \\
\mathbf{0 . 0 1 2}\end{array}$ \\
\hline $\begin{array}{c}\text { Antibiotic Class prescribed, n (\%) } \\
\text { Cephalosporin } \\
\text { Penicillin } \\
\text { Macrolide } \\
\text { Fluoroquinolone } \\
\text { Other }\end{array}$ & $\begin{array}{c}4,(44) \\
16,(16) \\
8,(8) \\
8,(8) \\
24,(24)\end{array}$ & $\begin{array}{l}24,(24) \\
17,(17) \\
9,(9) \\
17,(17) \\
33,(33)\end{array}$ & $\begin{array}{l}\mathbf{0 . 0 1 5} \\
0.861 \\
0.808 \\
0.071 \\
0.233\end{array}$ \\
\hline
\end{tabular}

$\mathrm{SD}=$ standard deviation

Table 2. Main outcomes

\begin{tabular}{|c|c|c|c|}
\hline Readmission Type & Pre-intervention (N=100) & Post-intervention (N=100) & P value \\
\hline 30-day all-cause readmission rates, days, $n(\%)$ & $28,(28)$ & $28,(28)$ & $4,(4)$ \\
\hline 30-day readmission rates due to infection, $\mathrm{n}(\%)$ & $2,(2)$ & 6.414 & $6.6 \pm 4.4$ \\
\hline Length of stay, day, (mean, \pm SD) & $9.0 \pm 6.5$ & $\mathbf{0 . 0 0 3}$ \\
\hline
\end{tabular}

$\mathrm{SD}=$ standard deviation

undergoing clean or clean-contaminated surgeries in cardiothoracic departments is associated with a significantly shorter length of stay compared to patients treated during the pre-intervention period $(P<0.05)$.

Another interesting observation was a significant decrease in the prescription of cephalosporins. This finding can be attributed to multiple factors, such as the increased prevalence of gram-negative infection and urinary tract infections, making treatment with cephalosporin less preferable than fluoroquinolone. In addition, prescribers' preferences can impact the decreased use of cephalosporin. Some prescribers in our institution might find that cephalosporins require high frequency of administration, which can decrease patient compliance. Moreover, due to the retrospective nature of the study, selection bias could lead to the decreased use of cephalosporins.

In the current study, pharmacist led antimicrobial stewardship intervention showed minimal effect on readmission due to infection because of the low prevalence of infection in patient with mental health disorders. This finding was supported by Heanen, et al. [2] They conducted a study in which surveys were completed by physicians and/or nurses to evaluate the prevalence of infections in psychiatric patients. Infection was defined according to the CDC guidelines, and psychiatric disorders included those listed in the "Diagnoses and Statistical Manual of Mental Disorder III Revised" (DSM IIIR). Of the 8,679 patients included in the study, they found that $13.1 \%$ of patients had at least one infection, while $3.3 \%$ of the patients were treated with antibiotics. Hence, it can be inferred that the antimicrobial stewardship intervention plays least role in the readmission due to infection.

The current study has several limitations. First, the study was conducted in a single tertiary academic medical center, so it is unknown if patients were admitted to another facility during the 30-day postdischarge. Second, it is unknown if discharged patients completed full courses of antibiotics. During the hospital stay, the use of antimicrobial regimen was documented for each patient in the behavioral health units. However, it was not feasible to document patient compliance post-discharge. Third, the antimicrobial stewardship intervention was only conducted on one day per week, during which a pharmacist would run a report that included all patients from behavioral health units who were currently receiving antibiotic therapy. However, as the average length of stay in our behavioral health units is 6-7 days, the likelihood of missing patients is low. Fourth, the lack of similar studies makes it difficult to estimate the required sample size. The current study could be underpowered, which may have contributed to the insignificant differences in primary and secondary outcomes.

\section{Conclusion}

In conclusion, a pharmacist-led antimicrobial stewardship intervention did not affect all-cause and infection-related readmission rates within 30 days of discharge in patients admitted to behavioral health units and prescribed oral antimicrobial therapy. However, there was a significantly shorter hospital stay in the post-intervention group. Future studies are warranted to gain clearer insight into the effect of antimicrobial stewardship intervention on the readmission rate of patient with mental health disorders.

\section{References}

1. Jencks SF, Williams MV, Coleman EA (2009) Rehospitalizations among patients in the Medicare fee-for-service program. $N$ Engl J Med 360: 1418-1428. [Crossref]

2. Haenen R, Moens G, Jacques P (1997) The prevalence of infections in psychiatric institutes in Belgium. J Hosp Infect 37: 273-280. [Crossref]

3. Nilholm H, Holmstrand L, Ahl J, Månsson F, Odenholt I, et al. (2015) An Audit-Based, Infectious Disease Specialist-Guided Antimicrobial Stewardship Program Profoundly Reduced Antibiotic Use Without Negatively Affecting Patient Outcomes. Open Forum Infect Dis 2: ofv042.

4. Bohan JG, Remington R, Jones M, Samore M, Madaras-Kelly K (2017) Outcomes Associated with Antimicrobial De-escalation of Treatment for Pneumonia Within the Veterans Healthcare Administration. Open Forum Infect Dis 4: ofw244.

5. Ahmedani BK, Solberg LI, Copeland LA, Fang-Hollingsworth Y, Stewart C, et al (2015) Psychiatric comorbidity and 30-day readmissions after hospitalization for hear failure, AMI, and pneumonia. Psychiatr Serv 66: 134-140. 
6. Burke RE, Donzé J, Schnipper JL (2013) Contribution of psychiatric illness and substance abuse to 30-day readmission risk. J Hosp Med 8: 450-455. [Crossref]

7. Seah VXF, Ong RYL, Lim ASY, Chong CY, Tan NWH, et al. (2017) Impact of a Carbapenem Antimicrobial Stewardship Program on Patient Outcomes. Antimicrob Agents Chemother 61(9). [Crossref]

8. Gupta K, Hooton TM, Naber KG, Wullt B, Colgan R, et al. (2011) International clinical practice guidelines for the treatment of acute uncomplicated cystitis and pyelonephritis in women: A 2010 update by the Infectious Diseases Society of America and the European Society for Microbiology and Infectious Diseases. Clin Infect Dis 52: 103.
9. Stevens DL, Bisno AL, Chambers HF, Dellinger EP, Goldstein EJC, et al. (2014) Practice guidelines for the diagnosis and management of skin and soft tissue infections: 2014 update by the infectious diseases society of America. Clin Infect Dis 59: 147-159.

10. Mandell LA, Wunderink RG, Anzueto A, Bartlett JG, Campbell GD, et al. (2007) Infectious Diseases Society of America/American Thoracic Society consensus guidelines on the management of community-acquired pneumonia in adults. Clin Infect Dis 44 Suppl: 27.

11. Zhou L, Ma J, Gao J, Chen S, Bao J (2016) Optimizing Prophylactic Antibiotic Practice for Cardiothoracic Surgery by Pharmacists' Effects. Medicine (Baltimore) 95: e2753.

Copyright: (C2017 Althagafi AA. This is an open-access article distributed under the terms of the Creative Commons Attribution License, which permits unrestricted use, distribution, and reproduction in any medium, provided the original author and source are credited. 